\title{
Comparison of transcriptome technologies in the pathogenic fungus Aspergillus fumigatus reveals novel insights into the genome and MpkA dependent gene expression
}

Sebastian Müller ${ }^{1}$, Clara Baldinn ${ }^{2,3}$, Marco Groth $^{4}$, Reinhard Guthke', Olaf Kniemeyer ${ }^{2,3,5}$, Axel A Brakhage $e^{2,3}$ and Vito Valiante $2^{2^{*}}$

\begin{abstract}
Background: The filamentous fungus Aspergillus fumigatus has become the most important airborne fungal pathogen causing life-threatening infections in immuno-compromised patients. Recently developed high-throughput transcriptome and proteome technologies, such as microarrays, RNA deep-sequencing, and LC-MS/MS of peptide mixtures, are of enormous value for systematically investigating pathogenic organisms. In the field of infection biology, one of the priorities is to collect and standardise data, in order to generate datasets that can be used to investigate and compare pathways and gene responses involved in pathogenicity. The "omics" era provides a multitude of inputs that need to be integrated and assessed. We therefore evaluated the potential of paired-end mRNA-Seq for investigating the regulatory role of the central mitogen activated protein kinase (MpkA). This kinase is involved in the cell wall integrity signalling pathway of $A$. fumigatus and essential for maintaining an intact cell wall in response to stress.
\end{abstract}

Results: The comparison of the transcriptome and proteome of an A. fumigatus wild-type strain with an mpkA null mutant strain revealed that $70.4 \%$ of the genome was found to be expressed and that MpkA plays a significant role in the regulation of many genes involved in cell wall remodelling, oxidative stress and iron starvation response, and secondary metabolite biosynthesis. Moreover, absence of the $m p k A$ gene also strongly affects the expression of genes involved in primary metabolism. The data were further processed to evaluate the potential of the mRNA-Seq technique. We comprehensively matched up our data to published transcriptome studies and were able to show an improved data comparability of mRNA-Seq experiments independently of the technique used. Analysis of transcriptome and proteome data revealed only a weak correlation between mRNA and protein abundance.

Conclusions: High-throughput analysis of MpkA-dependent gene expression confirmed many previous findings that this kinase is important for regulating many genes involved in metabolic pathways. Our analysis showed more than 2000 differentially regulated genes. RNA deep-sequencing is less error-prone than established microarray-based technologies. It also provides additional information in A. fumigatus studies and as a result is more suitable for the creation of extensive datasets.

Keywords: Aspergillus fumigatus, mRNA-Seq, Transcriptome, Proteome, Secondary metabolite gene clusters, Cell wall integrity pathway

\footnotetext{
* Correspondence: vito.valiante@hki-jena.de

${ }^{2}$ Department of Molecular and Applied Microbiology, Leibniz Institute for Natural Product Research and Infection Biology - Hans Knöll Institute, Beutenbergstr. 11a, Jena 07745, Germany

Full list of author information is available at the end of the article
} 


\section{Background}

The development of new transcriptome techniques, coupled with the ability of creating and analysing huge databases, has paved the way for system biology's "Golden Age". The generation and processing of highthroughput data makes possible the investigation of the genome, transcriptome, proteome and metabolome of living organisms in an overall context. This is particularly useful for gaining a deeper understanding of pathogenic microorganisms [1].

The above holds also true for Aspergillus fumigatus, which can be regarded as the most important airborne fungal pathogen. This fungus can cause a lifethreatening disease, invasive aspergillosis (IA), in immuno-compromised patients. Patients that suffer from IA still have a low life expectancy. This is due to the lack of reliable diagnostic tools and of efficient antifungal therapies [2,3].

Transcriptome analysis has just recently been applied to A. fumigatus. The first genome-sequencing project, applied to the isolate Af293 and coupled with the first transcriptome data available, has been published [4] and a second genome, from the A. fumigatus strain A1163, is now available [5]. In the last few years, various microarray platforms have been designed for global transcriptome analyses of A. fumigatus. These platforms have all been developed independently of each other using defined sets of primers and probes. However, developing microarray platforms for $A$. fumigatus has already been made outdated by the advent of new technologies based on cDNA sequencing.

To show the potential possibilities of mRNA-Seq to accelerate Aspergillus research and to deepen our knowledge about the regulatory function of MpkA, we analysed $A$. fumigatus wild-type and the corresponding mutant lacking the $m p k A$ gene by cDNA sequencing and $2 \mathrm{D}$ gel-based proteomics. The gene mpkA codes for the mitogen activated protein kinase MpkA which acts on the A. fumigatus cell wall integrity (CWI) signalling pathway [6,7]. The $\triangle m p k A$ mutant strain is sensitive to cell-wall active compounds, oxidative stress and heat shock. The function of this gene is also related to polyamine metabolism and to the iron depletion response [8]. Moreover, it affects the expression of several secondary metabolite gene clusters. The $\triangle m p k A$ mutant strain produces less gliotoxin than the wild type and this is a potent immunosuppressant belonging to the epipolythiodioxopiperazine class of fungal toxins [8,9]. Our transcriptomic and proteomics data also allowed us to compare different omics-techniques. Analysis of the mRNA sequences obtained revealed unexpected novelties in the A. fumigatus genome. We also found that $30 \%$ of the transcriptionally active $A$. fumigatus genome has not been annotated in the canonical genome databases (e.g. CADRE, Broad, and AspGD) [10,11].

In addition, we addressed the question of how the data obtained with these new technologies differ from publically available microarray data. To do this we compared published transcriptome studies carried out by using either microarrays or by applying mRNA-Seq with the mRNA-Seq dataset we generated. This comparison demonstrated that the data varies more among the different microarray platforms than between mRNA-Seq experiments. Additionally, all the collected transcriptome data were compared with proteome data. Although the data obtained by mRNA-Seq was better correlated with the $A$. fumigatus proteome, there was still a relatively low correlation between the two different datasets.

\section{Results}

mRNA-Seq data set summary

To gain a deeper insight into the regulatory circuits of the MAP-kinase MpkA in A. fumigatus we extracted RNA from a null $m p k A$ mutant and from the corresponding wild-type strain. We sequenced a total of six libraries, three biological replicates for the $\triangle m p k A$ strain and three for the wild-type strain. We obtained about 263 million paired end reads. Of these, 193 million (74\%) were uniquely mapped against the A. fumigatus A1163 genome. Using a stringent RPKM cut-off of 10 reads per gene, 8172 genes $(80 \%$ of the total annotated genes) were found to be transcribed in the wild-type strain we used. The coverage obtained was similar to that analysed in the dataset published by Gibbons et al. [12] for A. fumigatus (72\% of the genes having an RPKM greater than 10). It was also similar to an mRNA-Seq study in $A$. oryzae [13], where the majority (83.4\%) of the genome was covered by at least one read. Requiring coverage of at least 10 overlapping paired-end reads per base-pair, 70.4\% ( 20 million base pairs) of the genome was found to be expressed. This is remarkable, considering the fact that according to the A. fumigatus CADRE genome database, only $\sim 50 \%$ of the genome is potentially transcribable. These findings indicated that the A. fumigatus transcriptome has been greatly underestimated.

\section{UTRs prediction and annotation of new genes}

So far, most studies have used mRNA-Seq data to identify transcriptional islands, which are consecutive areas of overlapping reads [13]. We additionally checked these Transcript Active Regions (TARs) for open reading frames. Since this approach is error prone, we employed $a b$ initio gene prediction combined with mRNA-Seq data to improve the quality of gene prediction (Figure 1). This evidence-based gene prediction has the advantage over the classic $a b$ initio prediction of finding gene 


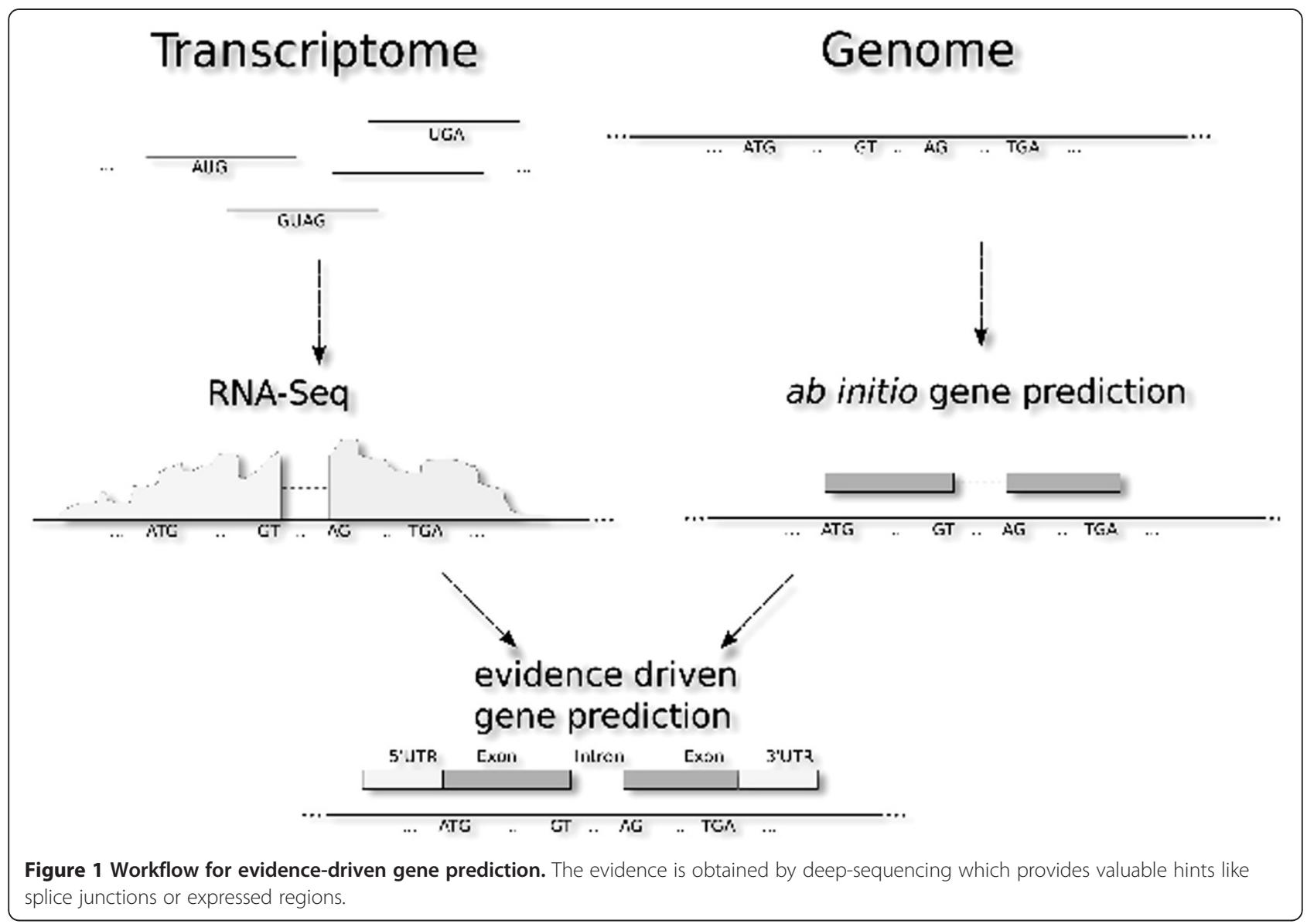

structures by making use of the mRNA-Seq expression profiles. This combination incorporates intron-junction information derived from intron-spanning reads and allows the prediction of not translated regions (UTR) in a systematic manner.

Gene prediction still requires manual work to find appropriate adjustments for integrating data within a classic gene-predicting framework. By using mRNA-Seq data, we identified 185 new transcripts coding for putative proteins (Additional file 1: Table S1 and Table S2). However, we could not exclude the possibility that the new transcripts are part of untranslated regions (UTRs) or even part of genes that need to be re-annotated. Among all the identified new transcripts, 44 of them have unknown functions, while 141 have orthologs in the genomes of other fungi.

Incorporating mRNA-Seq data into gene prediction also allowed the identification of UTRs (Additional file 1: Table S2). However, UTR prediction remains a difficult task as only expressed regions provide evidence for UTR length. The method can therefore result in vague length predictions, especially for genes with low expression, due to insufficient coverage. Nevertheless, the identification of 5'UTR can help to find upstream open reading frames (uORFs) and led in our case to the identification of one potential uORF among the newly identified genes (Additional file 1: Table S2). However, knowledge of UTR sequences in $A$. fumigatus remains poor. This is mainly due to the lack of available expressed sequence tag (EST) libraries. Our analysis highlighted the fact that some genes can have multiple or alternative transcriptional starting points. We found an average median length for 5'UTR of 308 and for 3'UTR of 97 nucleotides based on 9912 UTR sequences for each gene considered (Additional file 2: Figure S1).

\section{Comparison of transcriptome technologies}

To better evaluate the performance of microarrays and mRNA-Seq, differentially expressed transcripts were investigated. We compared transcript levels in both the wild-type strain and the $\triangle m p k A$ mutant strain by repeating the experiment performed by Jain et al. [8].

Transcriptome data obtained by mRNA-Seq identified 2046 differentially regulated genes ( $\log _{2}$ fold change $>2$ and likelihood $>0.99$ ). For comparison, using the Febit microarray platform, 653 differentially expressed genes were identified (fold change $>1.5$ ) (see Additional file 1: Table S3, Table S4, and Table S5). KEGG analysis 
showed that common gene categories were enriched by the differentially expressed genes identified in the two datasets (Figure 2 and Additional file 1: Table S6, Table S7, and Table S8). The main difference between data sets was that mRNA-Seq gave a more detailed picture. The number of enriched genes for a specific category was substantially higher showing, for example, complete secondary metabolite gene clusters to be differentially regulated (e.g. the gene cluster bordered by genes AFUB_000840 and AFUB_000750). It is noteworthy that genes enriched in the KEGG category 0.1.1., i.e. genes involved in metabolic pathways, were differentially regulated in the mRNA-Seq data, but in contrast, were not enriched in the data set obtained using microarrays. The majority of these genes, in particular genes putatively involved in fatty acid metabolism, were down-regulated in the $\triangle m p k A$ mutant. This suggests that the RNA deep sequencing technique is more sensitive in finding differentially expressed genes than the microarray technique. This might be explained by the greater dynamic range covered by the RNA technique $[14,15]$.

For a more detailed analysis, we computed log fold changes for the $\triangle m p k A$ mutant $v s$ the wild-type of the differentially expressed genes based on both microarray and mRNA-Seq data, including two biological replicates for each (Figure 3). Correlation of log fold changes of expressed genes was highest within the same technology. Correlation within mRNA-Seq replicates (Pearson's correlation coefficient $r=0.96$ ) was higher than that between the two microarray replicates $(r=0.53)$. This low correlation was mainly due to genes that only slightly changed their expression between conditions and are therefore more affected by the technological noise resulting from the technology used. A possible explanation is the low sensitivity of microarrays for low expressed genes that results in a smaller dynamic range compared to sequence data [15].

When comparing the global transcript fold changes between mRNA-Seq and microarrays the correlation amounted to 0.14-0.19 considering all expressed genes (Figure 3). This was not surprising because most genes are not expected to be differentially expressed, which consequently reduces the correlation. Since these genes are usually not of interest, we selected genes having the highest absolute fold change in both studies (10\% quantiles of the total number of genes, intersection from mRNA-Seq and microarrays). The correlation value for this subset resulted in a large increase of both the within-array correlation (from 0.53 to 0.83 ) and the cross-technology correlation (between mRNA-Seq and microarrays, from 0.19 to 0.41 ). However, the 0.41 correlation is still lower than those found in other studies

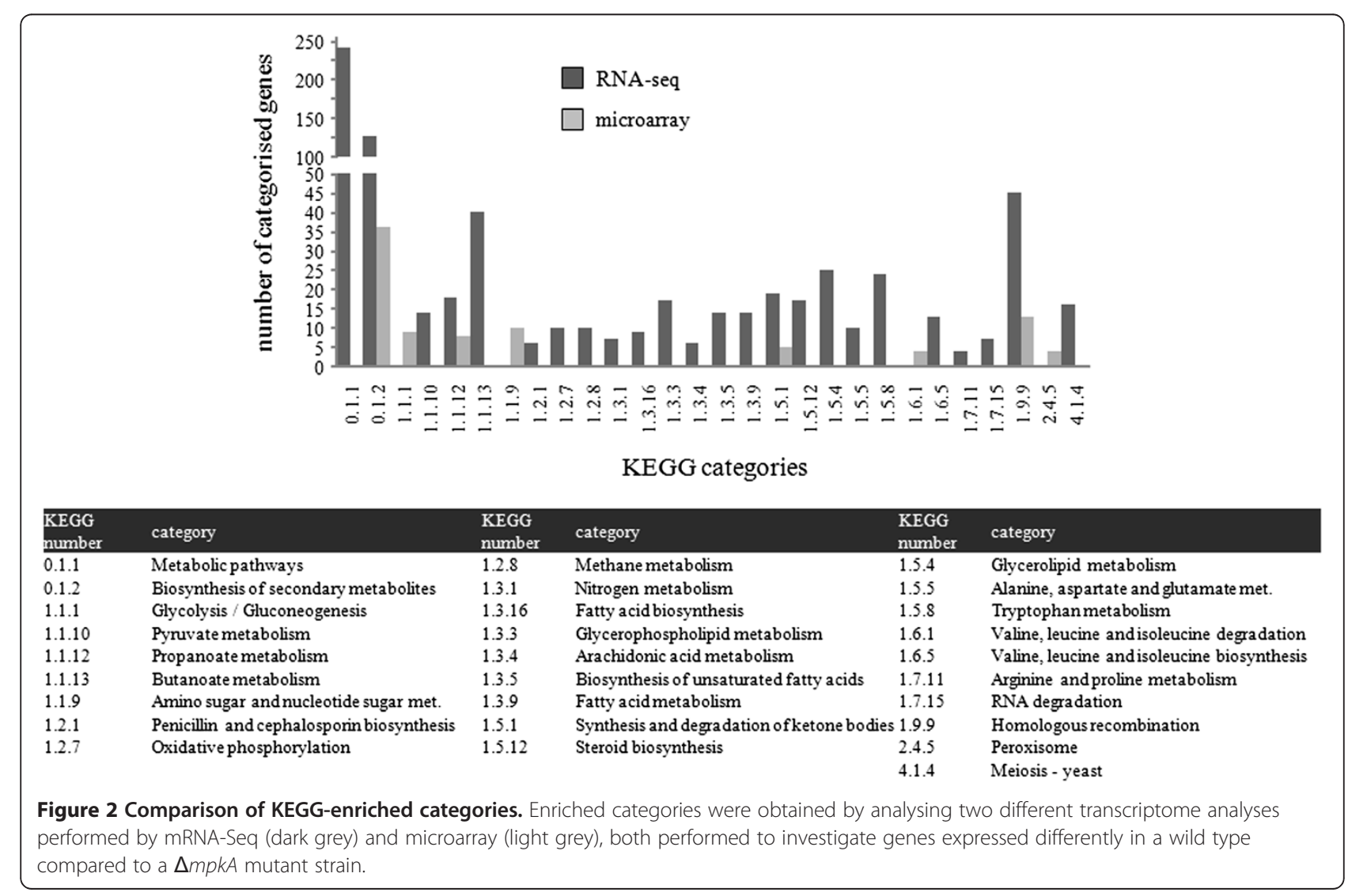




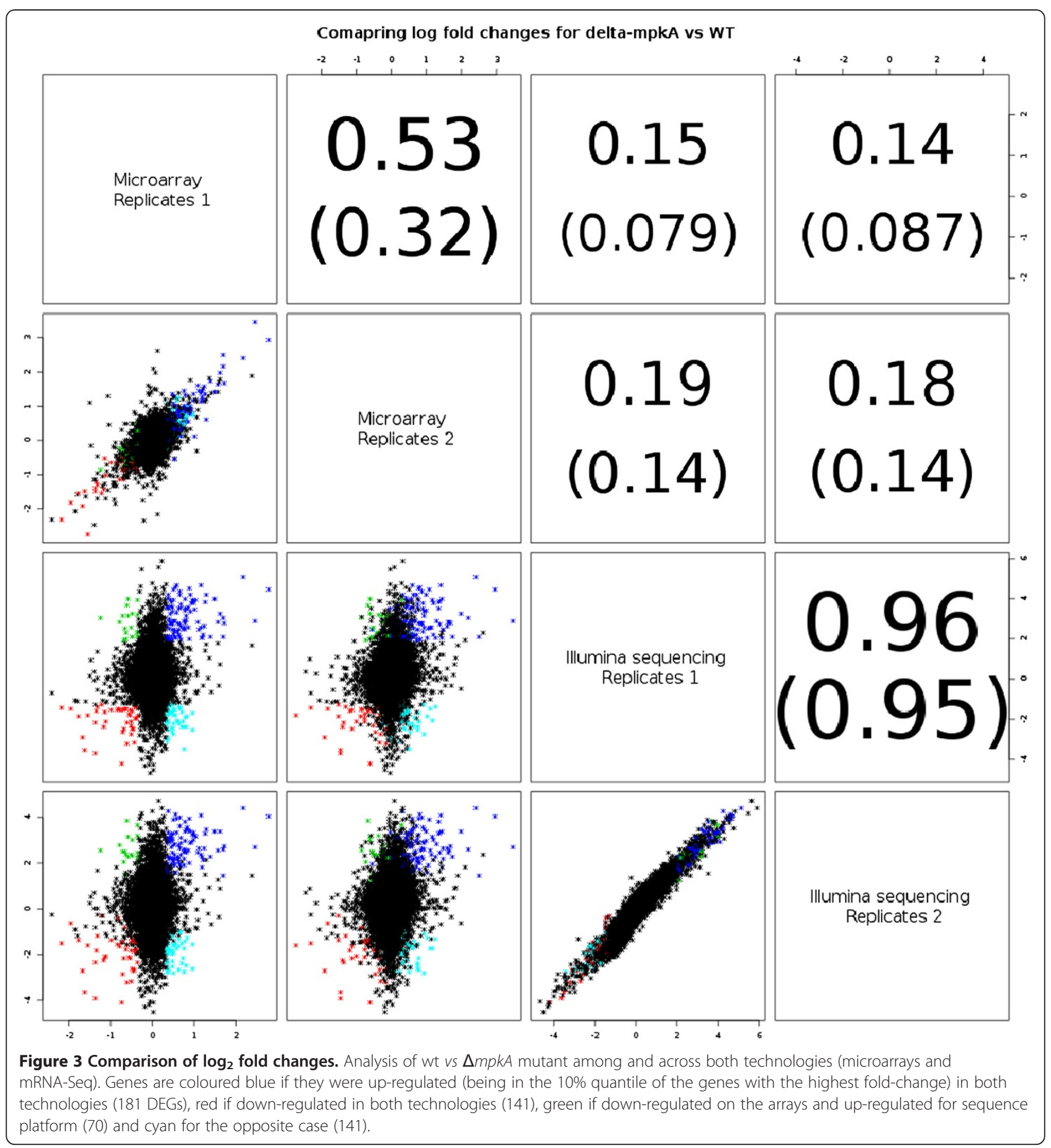

(e.g. Marioni et al. [16] found a correlation of 0.73 by comparing log fold changes between Affymetrix arrays and Illumina sequencing in experiments performed on human samples).

With the purpose of having additional values for evaluating the transcriptome data from the two technical platforms, qRT-PCR was performed in order to quantify transcript levels of genes that showed different expression levels in both studies (Figure 4, Additional file 1:
Table S9 and Additional file 3: Figure S2). Among the 15 transcripts investigated, 10 confirmed the direction of expression change detected by both transcriptome analysis techniques (with higher significance for mRNA-Seq data). Surprisingly, qRT-PCR did not reveal significant changes in some genes that showed opposite regulation in the analyses of the two techniques. For instance, qRTPCR confirmed the mRNA-Seq results for the sidD gene, coding for a non-ribosomal peptide synthetase involved 


\section{A}

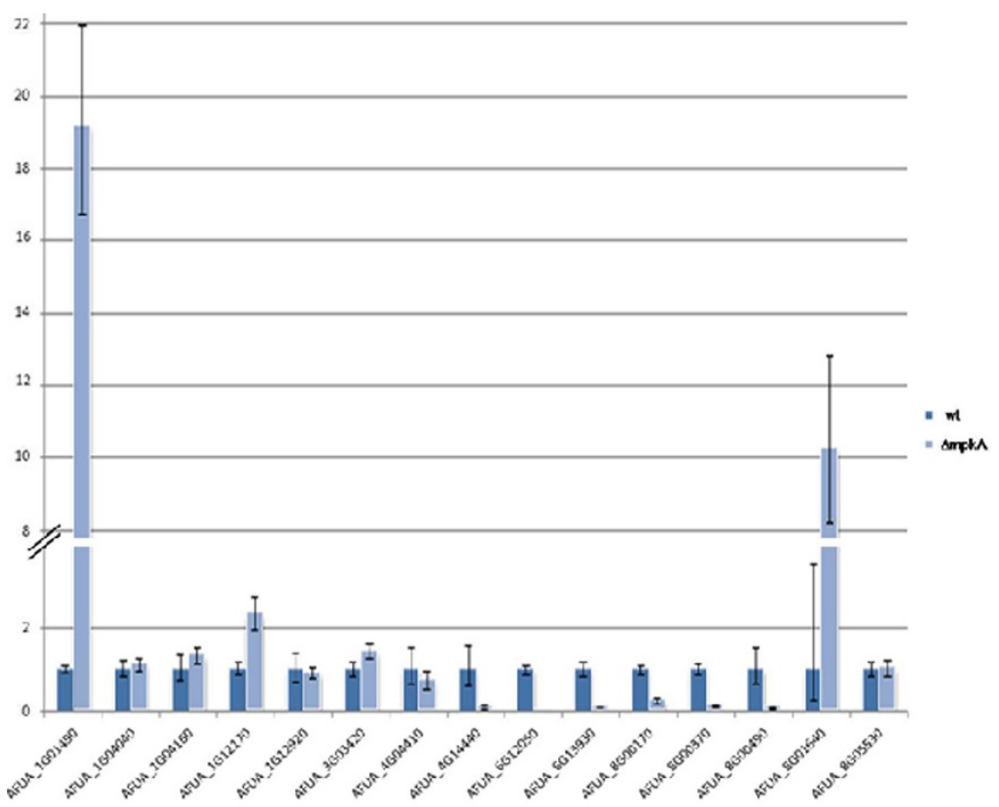

\begin{tabular}{|c|c|c|c|c|c|}
\hline Acc num & Acc num & name & Microarry & RNA-seq & 2D Dige \\
\hline AFU__1G01490 & AFUB_079300 & NACHT domain potein & 4,19 & 6,55 & NA \\
\hline AFU___ 1604040 & AFUB_0 04420 & RNA polymerase I & NA & $-1,47$ & $-3,06$ \\
\hline AFUA_1G04160 & AFUB_0 04510 & aspartate aminotransferase & 2,92 & $-3,95$ & $\mathrm{NA}$ \\
\hline AFUA_1G12170 & AFUB_011640 & elongation factor $\mathrm{FF}-\mathrm{Tu}$ & $-1,12$ & 2,65 & $-1,89$ \\
\hline AFUA_1G12920 & AFUB_012400 & glycogen phosphorylase GlpV/Gph1 & 3,09 & $-2,41$ & $\mathrm{NA}$ \\
\hline AFUA_3G03420 $_{3}$ & AFUB_044830 & nonibosomal peptide synthase SidD & $-2,09$ & 3,92 & NA \\
\hline AFUA_4G04410 & AFUB_098610 & 3-hydroxybutyryl-C aA dehydrogenase & 1,32 & $-2,77$ & 1,69 \\
\hline AFUA_4G14440 & AFUB_071690 & NRPS-like enzyme & $-1,06$ & 4,41 & NA \\
\hline AFUA_6G1 2050 & AFUB_078040 & nonibosomal peptide synthase & $-2,01$ & $-3,79$ & $\mathrm{NA}$ \\
\hline AFUA_6G1 3930 & AFUB_000820 & LovB-like polyketide synthase & $-1,36$ & $-2,49$ & NA \\
\hline AFUA_SG00170 & AFUB_0 86360 & nonibosomal peptide synthase FomA & $-1,40$ & $-6,26$ & NA \\
\hline AFUA_\&G00370 & AFUB_086200 & polyketide synthase & $-2,02$ & 4,85 & NA \\
\hline AFUA_SG00490 & AFUB_0 86080 & PKS-like enzyme & $-2,04$ & 4,81 & NA \\
\hline AFUA_SG01640 & AFUB_0 84950 & NRPS-like enzyme & 1,14 & 4,29 & $\mathrm{NA}$ \\
\hline AFUA_ $8 G 05530$ & AFUB_0 82020 & fumarate reductase $0 \mathrm{sm} 1$ & 1,00 & $-3,77$ & 35,26 \\
\hline
\end{tabular}

Figure 4 Differentially expressed gene and protein abundances. A) qRT-PCR used to check genes that showed differential expression between wt and $\triangle m p k A$ strains during microarray, mRNA-Seq and 2D proteome analysis. The relative amount of transcripts was normalised by setting the value for each wt gene to $1 ; \mathbf{B}$ ) table listing the genes investigated with their respective fold change ( $\Delta$ mpkA vs wt strains) during microarray, mRNA-Seq and 2D proteome analysis.

in siderophore production (AFUA_3G03420). Microarray data showed that genes expressed during low iron stress response were down-regulated in the $\triangle m p k A$ mutant strain. Previous experiments reversed microarray results, demonstrating that MpkA negatively regulates genes involved in siderophore production, indicating that many genes involved in iron depletion response are up-regulated in the $\triangle m p k A$ mutant strain (e.g. sidA or sidD) [8]. Current mRNA-Seq data confirmed our experimental hypothesis, by showing that genes involved in the iron starvation response are, in fact, up-regulated.

In order to study absolute transcript abundance relationships within and across various microarray and
mRNA-Seq platforms, we performed a comprehensive comparison. Analysis of transcriptome techniques included five microarray studies (three different platforms) and two different experiments performed using mRNA-Seq analysis (created in different labs) (Table 1). In this comparison, we included one representative sample corresponding to wild type for each experiment. For the study of Jain et al. [8] we also included two independent probe measurements in addition to the standard one (i.e., combined) to assess within-platform expression.

In total, the dataset compiled consists of seven wildtype measurements that were compared in a pair-wise 
Table 1 Arrays analysed

\begin{tabular}{|c|c|c|c|c|c|c|c|c|}
\hline $\mathrm{N}^{\circ}$ & Technique & Platform & Used strains & Growth conditions & Study & References & Accession & Technology \\
\hline 1 & Microarray & $\begin{array}{l}\text { TIGR } \\
(30 \mathrm{k} \text { v1) }\end{array}$ & Af293 & $\begin{array}{l}5 \times 10^{6} \text { conidia per } \mathrm{ml}, \\
\text { inoculated in complete } \\
\text { media, for } 17 \text { hours, } \\
\text { at } 30-37^{\circ} \mathrm{C}, 160 \mathrm{rpm}\end{array}$ & $\begin{array}{l}\text { Temperature shifts } \\
\left(30 \text { to } 37^{\circ} \mathrm{C} \text { and }\right. \\
30 \text { to } 48^{\circ} \mathrm{C} \text { ) }\end{array}$ & [4] & $\begin{array}{l}\text { ArrayExpress: } \\
\text { E-MEXP-332 }\end{array}$ & $\begin{array}{l}\text { DNA amplicon } \\
\text { microarray }\end{array}$ \\
\hline 2 & Microarray & $\begin{array}{l}\text { Custom } \\
\text { (Roche) }\end{array}$ & Af293 & $\begin{array}{l}10^{8} \text { conidia per ml, } \\
\text { inoculated in potato } \\
\text { dextrose media, for } \\
24 \text { hours, at } 28^{\circ} \mathrm{C} \\
160 \mathrm{rpm}\end{array}$ & $\begin{array}{l}\text { Effects on growth } \\
\text { in the presence of TSA }\end{array}$ & [18] & GEO: GSE19682 & $\begin{array}{l}\text { 60mer } \\
\text { oligonucleotide }\end{array}$ \\
\hline 3 & Microarray & Febit & CEA17 & $\begin{array}{l}10^{6} \text { conidia per ml, } \\
\text { inoculated in minimal } \\
\text { media, for } 16 \text { hours, } \\
\text { at } 37^{\circ} \mathrm{C}, 200 \mathrm{rpm}\end{array}$ & $\begin{array}{l}\text { Comparison among } \\
\text { wild type, } \Delta g p r C \text {, and } \\
\Delta g p r D \text { mutant }\end{array}$ & [25] & Omnifung & $\begin{array}{l}\text { in situ } \\
\text { oligonucleotide }\end{array}$ \\
\hline 4 & Microarray & TIGR (22 K v3) & ATTC 46645 & $\begin{array}{l}10^{7} \text { conidia per ml, } \\
\text { inoculated in Brian's } \\
\text { media, for } 14 \text { hours, } \\
\text { at } 37^{\circ} \mathrm{C}, 150 \mathrm{rpm}\end{array}$ & $\begin{array}{l}\text { Comparison between } \\
\text { planktonic and } \\
\text { biofilm growth }\end{array}$ & [17] & GEO: GSE19430 & $\begin{array}{l}\text { in situ } \\
\text { oligonucleotide }\end{array}$ \\
\hline 5 & Microarray & Febit & CEA17 & $\begin{array}{l}10^{6} \text { conidia per ml, } \\
\text { inoculated in minimal } \\
\text { media, for } 16 \text { hours, } \\
\text { at } 37^{\circ} \mathrm{C}, 200 \mathrm{rpm}\end{array}$ & $\begin{array}{l}\text { Comparison between } \\
\text { wild type and } \triangle m p k A \\
\text { mutant }\end{array}$ & [8] & Omnifung & $\begin{array}{l}\text { in situ } \\
\text { oligonucleotide }\end{array}$ \\
\hline 6 & mRNA-Seq & Illumina & $\begin{array}{l}\text { ATTC } 46645 \\
\text { Af293 CEA10* }\end{array}$ & $\begin{array}{l}10^{7} \text { conidia per ml, } \\
\text { inoculated in Brian's } \\
\text { media, for } 14 \text { hours, } \\
\text { at } 37^{\circ} \mathrm{C}, 150 \mathrm{rpm}\end{array}$ & $\begin{array}{l}\text { Comparison between } \\
\text { planktonic and } \\
\text { biofilm growth }\end{array}$ & {$[12]$} & On enquiry & GAllx \\
\hline 7 & mRNA-Seq & Illumina & CEA17 & $\begin{array}{l}10^{6} \text { conidia per ml, } \\
\text { inoculated in minimal } \\
\text { media, for } 16 \text { hours, } \\
\text { at } 37^{\circ} \mathrm{C}, 200 \mathrm{rpm}\end{array}$ & $\begin{array}{l}\text { Comparison between } \\
\text { wild type and } \\
\Delta m p k A \text { mutant }\end{array}$ & This study & $\begin{array}{l}\text { ArrayExpress: } \\
\text { E-MTAB-1236 }\end{array}$ & GAllx \\
\hline 8 & Proteomic & 2D-DIGE & ATTC 46645 & $\begin{array}{l}10^{6} \text { conidia per ml, } \\
\text { inoculated in minimal } \\
\text { media, for } 16 \text { hours, } \\
\text { at } 37^{\circ} \mathrm{C}, 200 \mathrm{rpm}\end{array}$ & $\begin{array}{l}\text { Design the } A \text {. fumigatus } \\
\text { mycelial and } \\
\text { mitochondrial proteome } \\
\text { map }\end{array}$ & {$[21]$} & Omnifung & MALDI-TOF/TOF \\
\hline 9 & Proteomic & 2D-DIGE & CEA17 & $\begin{array}{l}10^{6} \text { conidia per ml, } \\
\text { inoculated in minimal } \\
\text { media, for } 16 \text { hours, } \\
\text { at } 37^{\circ} \mathrm{C}, 200 \mathrm{rpm}\end{array}$ & $\begin{array}{l}\text { Comparison between } \\
\text { wild type and } \triangle m p k A \\
\text { mutant }\end{array}$ & This study & On enquiry & MALDI-TOF/TOF \\
\hline
\end{tabular}

GEO: gene expression omnibus (www.ncbi.nlm.nih.gov/geo); Omnifung (www.omnifung.hki-jena.de); ArrayExpress (http://www.ebi.ac.uk/arrayexpress/). *CEA17 strain is the uracile auxotroph derived from the CEA10 strain.

manner. A pair wise scatter plot was realised by computing the Pearson and Spearman correlations for all pairs (Figure 5 and Additional file 1: Table S10 and Table S11). Spearman is a rank correlation technique that can capture some non-linear relationships and is less prone to outliers produced by highly abundant genes. As for Marioni et al. [16] and Feng et al. [14] the correlation between replicates for the same experiment ranged from 0.93 for the dataset of Bruns et al. [17] to 0.99 for the both mRNA-Seq studies, indicating high platformreproducibility for microarrays as well as for sequencing data (data not shown). Therefore, it was sufficient to include only one representative replicate for each platform to obtain concise comparisons.

The transcriptome data reported showed that expression values measured for transcripts were strongly scattered. Cross platform correlation was quite low, ranging from 0.12 ([17] vs [18]) to 0.52 ([4] vs [18]). Correlation between the independent measurements (probes $\mathrm{A}$ and B) as calculated for the dataset of Jain et al. [8] was only 0.64 , although all probes were exposed to the same samples. This indicates a strong technological bias of microarray data due to variation in hybridisation. At the same time, the correlation between the two Febit datasets for the combined probe measurements was quite high (0.89) pointing to a high conservation of technological bias in microarray platforms. In order to perform a general comparison between technologies, i.e., microarrays vs mRNA-Seq, the correlation of data within the same technology was used as a reference for the correlations between technologies. The highest correlation (0.68) among comparisons of mRNA-Seq data to microarray data was higher than the highest correlation between arrays (0.52, Roche vs. TIGR v1). This points to the fact 


\begin{tabular}{|c|c|c|c|c|c|c|c|c|c|}
\hline & 78,910 & & 5678,1011 & & 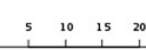 & & $\begin{array}{llll}2 & 2 \\
\end{array}$ & & \\
\hline & 0.64 & 0.95 & 0.91 & 0.33 & 0.10 & 0.26 & 0.32 & & 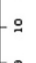 \\
\hline $\begin{array}{l}\text { Jan et al. } \\
\text { probes A }\end{array}$ & $(0.64)$ & & $(0.91)$ & $(0.32)$ & $(0.062)$ & $(0.25)$ & $(0.30)$ & $(0.30)$ & - \\
\hline$\therefore-1$ & & & 0.65 & 0.41 & 0.14 & 0.32 & 0.41 & 0.38 & \\
\hline & & $(0$ & (0. & $(0.39)$ & $(0.09)$ & $(0.30)$ & $(0.38)$ & $(0.37)$ & \\
\hline & & & & 0.37 & 0.13 & 0.30 & 0.37 & 0.35 & $\therefore$ \\
\hline & & combined & $(0$. & $(0.37)$ & $(0.078)$ & $(0.29)$ & $(0.35)$ & $(0.35)$ & $\because$ \\
\hline & & & & & 0.12 & 0.33 & 0.43 & 0.37 & \\
\hline & & & & $(0.42)$ & $(0.076)$ & $(0.33)$ & $(0.41)$ & $(0.37)$ & \\
\hline & & & & & 0.12 & 0.52 & 0.68 & 0.51 & $\because$ \\
\hline & & & & $\begin{array}{l}\text { Nishn } \\
\text { et }\end{array}$ & $(0.064)$ & $(0.52)$ & $(0.68)$ & $(0.52)$ & - \\
\hline & & & & & $\begin{array}{c}\text { TIGR v3 } \\
\text { Bruns } \\
\text { et al. }\end{array}$ & $\begin{array}{c}0.12 \\
(0.076)\end{array}$ & $\begin{array}{c}0.22 \\
(0.14)\end{array}$ & $\begin{array}{c}0.20 \\
(0.15)\end{array}$ & \\
\hline & & & & & & $\begin{array}{l}\text { TIGR v1 } \\
\text { Nierman } \\
\text { et al. }\end{array}$ & $\begin{array}{c}0.54 \\
(0.53)\end{array}$ & $\begin{array}{c}0.53 \\
(0.54)\end{array}$ & $\begin{array}{l}= \\
= \\
= \\
=\end{array}$ \\
\hline & & & & & & & $\begin{array}{c}\text { RNA-Seq } \\
\text { Gibbons } \\
\text { et al. }\end{array}$ & $\begin{array}{c}0.65 \\
(0.65)\end{array}$ & \\
\hline & & & & & & & & $\begin{array}{l}\text { RNA-Seq } \\
\text { This study }\end{array}$ & \\
\hline \multicolumn{10}{|c|}{$\begin{array}{l}\text { Figure } 5 \text { Pair-wise scatterplot of transcriptome datasets. Pair-wise scatterplot of the absolute RNA expression values measured by several } \\
\text { microarray platforms as well as mRNA-Seq: upper triangle, the pair-wise scatterplots; lower triangle, the corresponding Pearson correlation } r \\
\text { (Spearman correlation coefficient } r_{s} \text { in brackets) for each pair. A high correlation (with the maximum being one) indicates great agreement of } \\
\text { expression between the datasets compared. The green (red) coloured dots correspond to genes that are highly (little) expressed in the } \\
\text { mRNA-Seq data and little (highly) expressed in the microarray study of Jain el al. (2011) to check for bias due to the different technologies. }\end{array}$} \\
\hline
\end{tabular}

that RNA sequencing data are less affected by technological bias, which is confirmed by the scatter plot comparing both mRNA-Seq datasets (Figure 5).

Comparison between A. fumigatus transcriptome and proteome

In order to analyse a possible correlation between transcripts and protein abundances as well as fold-changes,
mRNA-Seq data, microarray data and the proteome data of the $A$. fumigatus wild-type and $\triangle m p k A$ mutant strain were compared. Looking at differential expression, 48 different proteins were identified by 2D-gel electrophoresis and subsequent MALDI-TOF / TOF, which showed a significant fold change in abundance (employing DIGE) in the $\triangle m p k A$ mutant compared to wild type. Gene enrichment and pathway analysis revealed that 
abundance of many proteins involved in metabolic processes and in oxidation/reduction processes is affected by MpkA (Additional file 1: Table S12 and Additional file 4: Figure S4).

The regulation of protein levels was also compared with transcriptome data obtained from microarrays developed by Febit and used in the study of Jain et al. [8] and our mRNA-Seq data. As in earlier results (Figure 3, based on 9800 genes rather than 48) the fold change correlation between microarrays and mRNA-Seq was low $(r=0.14$, Additional file 5: Figure S3). The measurements for transcriptome fold change often display low reproducibility because of difficulties in capturing the "true" fold change. For this reason, a low correlation between proteome and transcriptome technologies was assumed. However, the correlation between data obtained by microarrays and the proteome data was significantly higher $(r=0.28)$ than the correlation between mRNA-Seq data $v s$ proteome $(r=0.19)$. As in the comparative analysis of the transcriptome data, the low correlation applies to all genes with a low fold change resulting in a higher relative impact of technological noise. Furthermore, it has already been shown by studies on human and mouse $[19,20]$ that the relationship between transcriptome and proteome data is quite complex and protein levels are greatly influenced by post-translational processing and inherent variations in stability. These factors result in low correlations.

In order to study absolute protein abundances, we reanalysed the $A$. fumigatus 2D proteome map generated by Vödisch et al. [21], resulting in the identification of 312 unique proteins. These proteins were also present in the different transcriptome data mentioned above (Additional file 1: Table S13 and Table S14). In A. fumigatus, the relative abundances of the proteome map correlated surprisingly well with mRNA-Seq expression levels $(r=0.36$ for our data, and $r=0.5$ for data from [12], Figure 5) as well as with most microarrays, in particular the Febit arrays $(r=0.47-0.49)$. It is also noteworthy that the genes on the scatter plots (Figure 6) are more equally distributed for mRNA-Seq $v s$ proteome comparison (left bottom corner) whereas, in particular for Febit arrays, a bias was observed towards genes that are highly expressed on the arrays but with low expression on the proteome-map. This is most probably due to cross-hybridisation. By contrast, this sort of bias was not observed in the NGS data.

To check for genes that are potentially subject to posttranscriptional regulation, we selected genes that were very abundant in the mRNA-Seq data and rare in the proteome-map (coloured green in Figure 6) or the inverse (coloured red). FunCat-analysis using FungiFun tools [22] indicated that the "green" genes (i.e. those genes that are highly expressed according to mRNA-Seq but little expressed according to proteome data) were not significantly enriched within a functional category (even after multiple correction), whereas the red ones (i.e. those genes that are little expressed according to mRNASeq but highly expressed according to microarray data) were over-represented within the tricarboxylic-acid pathway and amino acid metabolism. These processes constantly require large amounts of proteins that are therefore less subjected to transcriptional regulation.

\section{Discussion}

Over recent years, it has become evident that the eukaryotic regulatory machinery linking cause (for instance, stress) to effect (stress response) is very complex. It usually involves the interplay of several regulatory layers such as transcriptome, epigenome, and proteome that might be subject to independent regulatory mechanisms. To understand these interactions as a whole, integrated studies are required.

We found that about $30 \%$ of the A. fumigatus genome expressed is located in assigned intergenic and intronic regions. This is most likely due to the presence of untranslated regions (UTRs) and transcripts that have not been classified so far. We investigated the potential of RNA deep-sequencing data to identify these genes by combining gene prediction (using the Augustus platform) and paired-end reads. This pioneer methodology helped us find almost 200 new transcripts with coding potential. Blast analysis revealed that more than half of these new transcripts harbour at least one ortholog in other Aspergillus species. This indicates the great potential of this methodology to find new genes and to improve gene annotations.

We tested RNA deep-sequencing to further investigate the role of MpkA in A. fumigatus. Previous studies demonstrated that this kinase is decisive for cell wall integrity signalling pathway regulation ([7]; 2010). Further studies revealed that MpkA is also involved in the regulation of the oxidative stress response, secondary metabolite production and siderophore biosynthesis during iron starvation [8]. Concerning the role of MpkA in $A$. fumigatus, mRNA-Seq data confirmed previous findings. Additionally, many genes involved in primary and secondary metabolism were affected by MpkA. In particular, genes putatively involved in amino acid biosynthesis were differentially expressed in the $\triangle m p k A$ mutant strain. This finding explained the strong difference found in the soluble amino acid pool composition for this strain [8]. Moreover, almost $10 \%$ of genes differentially expressed in the $\triangle m p k A$ mutant are putatively involved in primary metabolism (e.g. fatty acid metabolism and amino acid metabolism). These results confirmed that 


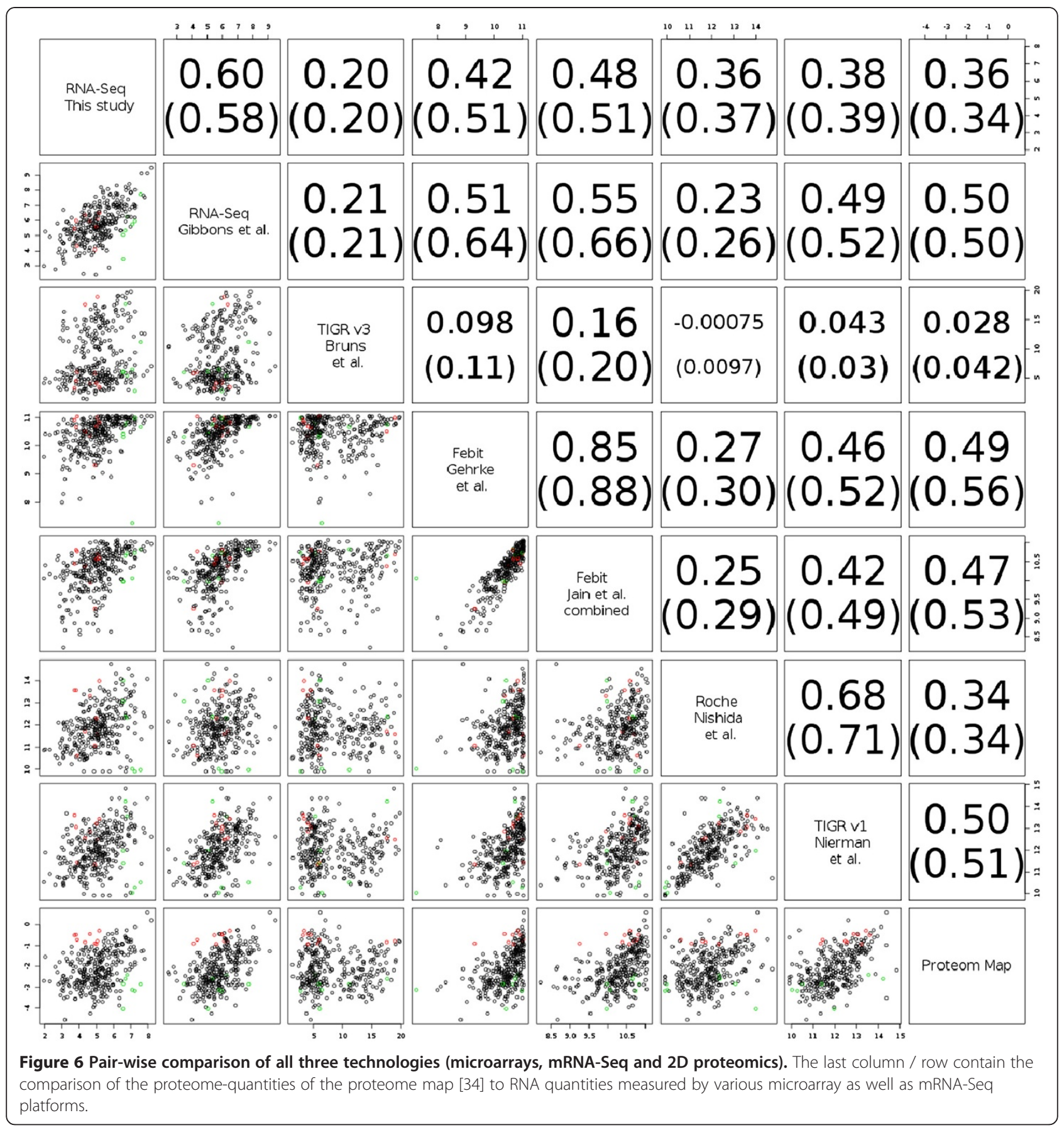

one of the functions carried out by MpkA is to fine-tune the balance between stress response and energy consuming cellular processes. Considering the genes involved in the iron-deficiency stress response, it was found that different techniques gave opposite results. It was previously reported that MpkA is important for regulating siderophore production by measuring an increased amount of siderophores in the $\triangle m p k A$ mutant strain during iron starvation [8]. This illustrates that mRNA-Seq is more reliable than other transcriptome techniques. However, the differences found between mRNA-Seq and qRT-PCR showed that it would be good practice if results obtained by sequencing were confirmed by applying ad hoc experiments.

Microarray data showed that genes belonging to several $A$. fumigatus secondary metabolite biosynthesis gene clusters were differentially expressed in the $\triangle m p k A \mathrm{mu}-$ tant (e.g. gliotoxin and pseurotin A). Secondary metabolite biosynthesis gene clusters are normally characterised by the presence of polyketide synthases (PKSs) or non- 
ribosomal peptide synthetases (NRPSs). Analysis of the A. fumigatus A1163 genome revealed the presence of 17 NRPSs, 13 PKSs and three hybrid NRPS/PKS enzymes [23].

Among them, 11 gene clusters have been found to be differentially regulated in the $\triangle m p k A$ mutant. Furthermore, in comparison with the microarray studies, the higher sensitivity of the mRNA-Seq technique led to the identification of almost all genes of a cluster to be differentially regulated. The mRNA-Seq approach is therefore superior to microarrays for studies on the global regulation of secondary metabolite biosynthesis.

The second question that we addressed concerned the comparability of mRNA-Seq data with published transcriptome data. We compared different technologies to investigate the A. fumigatus transcriptome (microarrays, mRNA-Seq and qRT-PCR). Information obtained by analysing these technologies were compared with a comprehensive set of proteome data obtained by 2D DIGE analysis followed by MALDI-TOF-MS / MS-based protein detection. The comprehensive dataset compiled in this study constitutes a basis for future studies with the aim of shedding light on the relationship between proteome and transcriptome data, in order, for example, to understand better post-transcriptional regulation on a global scale.

With regard to A. fumigatus, the results of whole genome transcriptome analyses based on microarrays were highly dependent on the platform used. Low consistency of microarray gene expression data can have multiple reasons, such as platform effects [24], different media, RNA extraction protocols, or biological variability. However, hybridisation seems to have the most significant impact. Hybridisation strongly depends on the probes, which were used to develop the microarrays. In the case of $A$. fumigatus, they have been designed independently from each other for the different platforms. The hybridisation bias seems to be highly conserved within the same platform, as shown by the high level of agreement between the studies of Jain et al. [8] and Gehrke et al. [25]. When considering the comparison between Jain et al. [8] microarray data with the mRNA-Seq data produced in this study, the correlation is relatively low $(r=0.35)$. Moreover, comparing the two datasets obtained with the same experimental proceeding by Bruns et al. [17] using microarrays and by Gibbons et al. [12] using mRNA-Seq, once again showed quite a low correlation $(r=0.22)$. The global comparison demonstrated that a higher correlation was found between the two mRNA-Seq datasets. This finding demonstrates that mRNA-Seq gives the highest level of comparability in terms of gene expression levels in A. fumigatus.

Comparative analysis of the A. fumigatus intracellular 2D proteome map from Vödisch et al. [21] revealed that protein abundances correlated well with transcriptome abundances measured with both microarray $(r=0.34$ $0.50)$ and mRNA-Seq techniques $(r=0.36-0.50)$. These values were higher than the ones observed by Albrecht et al. [26], who compared changes in A. fumigatus microarray and proteome data after heat shock induction. Previously, Foss et al. [27] calculated a correlation between proteome and transcriptome of $\mathrm{r}=0.186$ in Saccharomyces cerevisiae based on 278 proteins detected by LC-MS / MS analysis. Another study performed by Ghazalpour et al. [19] using LC-MS / MS-based quantifications of 486 mouse proteins, resulted in an average correlation of $\mathrm{r}=0.27$ (using Affymetrix microarray). However, although the results showed a better correlation between $A$. fumigatus proteome and transcriptome data, there was still a relatively low correlation between the two different datasets. This is most likely caused by a combination of several biases based on technological and biological effects.

\section{Conclusions}

The potential of next generation sequencing to investigate the regulation of the A. fumigatus transcriptome was demonstrated. We found that the transcriptional potential of A. fumigatus was underestimated. Almost 70\% of the genome was found to be actively transcribed. Sequence information obtained by mRNA-Seq was also used for gene prediction. We showed that the incorporation of transcriptome-based assembly can be very helpful for improving or confirming gene annotation. This methodology allowed us to identify 185 new transcripts never reported in the A. fumigatus A1163 strain. Compared to microarray data, we identified three times more differentially regulated genes in the $\triangle m p k A$ mutant strain compared with the wild type. Comparative transcriptome and proteome studies pointed out that MpkA plays an important role not only in maintaining the cell wall structure under stress condition but also in affecting genes involved in primary and secondary metabolism.

We compared data obtained by mRNA-Seq with those obtained by established microarray-based technologies for expression profiling. mRNA-Seq was found to be less error-prone and more suitable for the realisation of extensive datasets that can be potentially created by different groups under different lab conditions. Consequently, data produced so far using different microarray platforms, can only be considered by focusing on the highly differentially expressed genes.

\section{Methods}

\section{cDNA library construction and sequencing}

The A. fumigatus strain CEA17 ${ }^{\mathrm{KU} 80}$ strain was used for all experiments. The CEA17 ${ }^{\mathrm{KU} 80}$ strain was derived from the CEA17 wild-type strain [28]. 
Total RNA was isolated from wild-type and $\triangle m p k A$ strains cultured in Aspergillus minimal medium (AMM) for $16 \mathrm{~h}$ at $37^{\circ} \mathrm{C}$ [8], using the Qiagen RNeasy Plant Mini kit (Qiagen, Germany), according to the manufacturer's instruction. Three biological replicates for each strain were collected. Total RNA was used for Illumina nextgeneration sequencing [29]. For library preparation $5 \mu \mathrm{g}$ of total RNA per sample were processed using Illumina mRNA-Seq sample prep kit (RS-100-0801) following the manufacturer's instruction. The cDNA libraries were sequenced using a Genome Analyser (GAIIx) in a paired-end approach with $2 \times 36$ cycles resulting in paired reads with a length of 36 nucleotides per read. Each library was sequenced on a single lane and ended up with around 30-40 million reads per sample. Sequence data were extracted in FastQ format and used for further analysis.

\section{Mapping and normalising of transcriptome reads}

All reads were mapped using TopHat 1.2.0 [30] against the $A$. fumigatus A1163 genome (which is a derivative of the $A$. fumigatus CEA17 strain) retrieved from Ensemble release 9 . Parameters were set according to preliminary investigations, using a minimum intron length of 30 and a maximum of 4000. In agreement with the protocol used, from preliminary mapping a mean inner distance between mate pairs of 307 was found with a standard deviation of 110 . To avoid multiple hits for a single read, the maximum multi-hit option was set to one.

Transcript expression levels were normalised by counting the number of reads per kilobase of exon region per million mapped reads (RPKM) [31].

To detect genes differentially expressed in the wild type and the $\triangle m p k A$ mutant, we applied the $\mathrm{R}$ package baySeq [32] which takes advantage of the three biological replicates for each condition. RPKM and differentially expressed genes were determined using the $A$. fumigatus A1163 genome annotated by CADRE as a reference.

Differentially expressed genes were categorised using GO enrichment, KEGG enrichment, and FunCat analysis using the FungiFun platform [22].

\section{UTRs prediction}

With the purpose of identifying putative new transcripts, we performed new gene annotation employing Augustus $a b$ initio prediction [33] combined with hints generated from the mRNA-Seq reads. We present a new annotation including UTRs for A. fumigatus A1163. These hints consisted of intron junctions that were identified by TopHat as well as exon-parts revealed with the IRanges package which is part of the Bioconductor collection. The workflow for new gene annotation is reported in Figure 1.

\section{Microarray data}

The transcriptome and proteome data analysed are summarised in Table 1. The datasets were obtained in a pre-processed state i.e. background-corrected and normalised. Since the different microarray platforms and even the different $A$. fumigatus genomes have used different Gene-IDs (e.g. accession numbers starting with AFUB for the A1163 strain, and accession numbers starting with AFUA for the Af293 strain), all data were mapped according to the AFUA annotation, using ensemble mapping, to achieve comparability. The data were imported and analysed in the R / Bioconductor software environment employing the packages biomaRt and Biostrings.

\section{Real-Time PCR}

The qRT-PCR experiments to determine the amounts of selected putatively differently expressed genes were performed with StepOnePlus Real-Time PCR System (Applied Biosystems), using myTaq HS Mix 2x (Bioline) and Evagreen (Biotium). For each transcript analysed more primers were tested in tandem, in order to obtain primer efficiency close to $100 \%$ in all cases. To validate primers, efficiency standard curves were realised considering seven serial dilutions of genomic DNA of $A$. fumigatus in three replicates. All the primers selected for the qRTPCR analysis are shown in Additional file 1: Table S15. The A. fumigatus actin1 gene (AFUA_6G04740) was chosen as the normalising gene. The cDNA was generated using RevertAid Premium (Fermentas). As negative controls, a vial was prepared without reverse transcriptase, and another one was prepared without cDNA.

\section{Assessing differential expression}

To correlate the mRNA-Seq data with the corresponding presence of proteins, the mRNA-Seq data set was compared with the proteome data published by Vödisch et al. [21]. For our study, the normalised protein spot abundances on the 2D gels were reanalysed using the most recent version of the software Delta 2D 4.3 (Decodon, Germany).

The DIGE (difference in-gel electrophoresis) technique was used to analyse protein samples of wild type and $\triangle m p k A$ mutant mycelium cultivated in AMM for $16 \mathrm{~h}$ at $37^{\circ} \mathrm{C}$. Twelve samples (three independent biological replicates and two technical replicates for each strain as well as one mixed internal standard), were labelled with CyDye minimal dyes according to the manufacturer's protocol with slight modifications (GE Healthcare BioSciences, Munich Germany). We then labelled $50 \mu \mathrm{g}$ total protein of each sample with 300 pmol of CyDye DIGE fluorophores (dissolved in dimethyl formamide). Samples obtained from the wild-type and from the $\triangle m p k A$ mutant strain were labelled with either $\mathrm{Cy} 3$ or 
Cy5. A pool of all six samples $(3 \times$ wt and $3 \times \Delta m p k A)$ was prepared, labelled with $\mathrm{Cy} 2$, and used as a global internal standard. We carried out 2D gel processing, spot screening and MALDI-TOF / TOF analysis with an ultrafleXtreme mass spectrometer (Bruker Daltonics, Germany), as previously described [34] with slight modifications. FlexControl 3.3 software was used for data collection and flexAnalysis 3.3 for spectra analysis and peak list generation (Bruker Daltonics, Germany). Peptide mass fingerprint (PMF) and peptide fragmentation fingerprint (PFF) spectra were submitted to the MASCOT 2.3 server (Matrix Science, U.K.) searching the recent version of the NCPInr database but restricted to the fungi. Mascot search parameters were the following: fixed modification of cysteine thiols to S-carbamidomethyl derivatives, variable methionine oxidation, up to one missed cleavage and a peptide mass tolerance of $100 \mathrm{ppm}$.

\section{Accession number}

The RNA-Seq data were deposited in ArrayExpress (ArrayExpress accession: E-MTAB-1236).

\section{Additional files}

\section{Additional file 1: Tables.xls (Excel file which includes all the cited supplementary tables).}

Additional file 2: Figure S1. Length distribution of untranslated regions (UTRs). The analysis was based on 9912 3' and 5' UTR sequences. The red vertical lines indicate the average median length of the $5^{\prime}$ UTRs (308) and the 3'UTRs.

Additional file 3: Figure S2. Comparison of delta_delta_Ct values obtained by qRT-PCR analysis (see also Additional file 1: Table S9). Values obtained by qRT-PCR analysis where compared to $\log _{2}$ fold changes (wt vs $\triangle m p k A$ ) obtained by microarrays and mRNA-Seq based on 14 genes. Both technologies seem to agree with the qRT-PCR-data with an average Pearson correlation of $r=0.75$ for microarrays and 0.55 for mRNA-Seq (Spearman correlation coefficient $r_{s}$ in brackets).

Additional file 4: Figure S4. 2D gel electrophoresis of protein extracts. Total proteins were extracted from A. fumigatus wild type (Cy5, green) and $\triangle m p k A$ mutant strain (Cy3, purple). Proteins were stained with the difference in gel electrophoresis (DIGE) labelling technique. Total proteins were separated in a $\mathrm{pH}$ gradient of 3-11 (nonlinear).

Additional file 5: Figure S3. Pair-wise scatterplot of all three technologies. Comparison of three different technologies that is 2D-DIGE proteomic, mRNA-Seq and microarray, to detect differentially expressed genes and proteins between the $\Delta m p k a$ strain and wild-type strain, based on log fold changes of 94 entries. The overall correlation was found to be low, especially between mRNA-Seq and proteomic, whereas the correlation between proteomic and microarray was higher.

\section{Competing interests}

The authors declare that they have no competing interests.

\section{Authors' contributions}

$W$ and $S M$ designed research. SM, MG, CB and OK performed research. SM, $R G, O K, A A B$, and $W$ analysed data and wrote the paper. All authors read and approved the final manuscript.

\section{Acknowledgements}

We thank Silke Steinbach for excellent technical assistance and Martin Vödisch for reanalysing the proteome-map data. SM was supported by the
International Leibniz Research School (ILRS: http://www.ilrs.hki-jena.de/) for Microbial and Biomolecular Interactions as part of the excellence graduate school Jena School for Microbial Communication (JSMC: http://www.jsmc. uni-jena.de/). W was supported by a grant from the Federal Ministry of Education and Research (BMBF) in the frame of ERA-NetPathoGenoMics and in part from a European Re-integration Grant (Proposal 248815 CellWallPath) founded by the European Framework Program. CB was supported by the "ARIADNE" Marie Curie Training Network.

None of the funding bodies had any role in study design, data collection and analysis, decision to publish, or preparation of the manuscript.

\section{Author details}

${ }^{1}$ Department of Systems Biology / Bioinformatics, Leibniz Institute for Natural Product Research and Infection Biology - Hans Knöll Institute, Beutenbergstr. 11a, Jena 07745, Germany. ${ }^{2}$ Department of Molecular and Applied Microbiology, Leibniz Institute for Natural Product Research and Infection Biology - Hans Knöll Institute, Beutenbergstr. 11a, Jena 07745, Germany. ${ }^{3}$ Department of Microbiology and Molecular Biology, Institute of Microbiology, Friedrich Schiller University Jena, Beutenbergstr. 11a, Jena 07745, Germany. ${ }^{4}$ Department of Genome Analysis, Leibniz Institute for Age Research - Fritz Lipmann Institute, Beutenbergstr. 11a, Jena 07745, Germany. ${ }^{5}$ Integrated Research and Treatment Center, Center for Sepsis Control and Care Jena, University Hospital (CSCC), Jena 07747, Germany.

Received: 18 May 2012 Accepted: 21 September 2012

Published: 2 October 2012

\section{References}

1. Horn F, Heinekamp T, Kniemeyer O, Pollmächer J, Valiante V, Brakhage AA: Systems biology of fungal infection. Front Microbiol 2012, 3:118.

2. Brakhage AA: Systemic fungal infections caused by Aspergillus species: epidemiology, infection process and virulence determinants. Curr Drug Targets 2005, 6:875-886.

3. Dagenais TRT, Keller NP: Pathogenesis of Aspergillus fumigatus in invasive aspergillosis. Clinic Microbiol Rev 2009, 22:447-465.

4. Nierman WC, Pain A, Anderson MJ, Wortman JR, Kim HS, Arroyo J, Berriman M, Abe K, Archer DB, Bermejo C, Bennett J, Bowyer P, Chen D, Collins M, Coulsen R, Davies R, Dyer PS, Farman M, Fedorova N, Fedorova N, Feldblyum TV, Fischer R, Fosker N, Fraser A, García JL, García MJ, Goble A Goldman GH, Gomi K, Griffith-Jones S, et al: Genomic sequence of the pathogenic and allergenic filamentous fungus Aspergillus fumigatus. Nature 2005, 438:1151-1156.

5. Fedorova ND, Khaldi N, Joardar VS, Maiti R, Amedeo P, Anderson MJ, Crabtree J, Silva JC, Badger JH, Albarraq A, Angiuoli S, Bussey H, Bowyer P, Cotty PJ, Dyer PS, Egan A, Galens K, Fraser-Liggett CM, Haas BJ, Inman JM, Kent R, Lemieux S, Malavazi I, Orvis J, Roemer T, Ronning CM, Sundaram JP, Sutton G, Turner G, Venter JC, et al: Genomic islands in the pathogenic filamentous fungus Aspergillus fumigatus. PLoS Genet 2008, 4:e1000046.

6. Valiante $V$, Heinekamp $T$, Jain R, Haertl A, Brakhage AA: The mitogenactivated protein kinase MpkA of Aspergillus fumigatus regulates cell wall signaling and oxidative stress response. Fungal Genet Biol 2008, 45:618-627.

7. Valiante $V$, Jain $R$, Heinekamp T, Brakhage AA: The MpkA MAP kinase module regulates cell wall integrity signaling and pyomelanin formation in Aspergillus fumigatus. Fungal Genet Biol 2009, 46:909-918.

8. Jain R, Valiante V, Remme N, Docimo T, Heinekamp T, Hertweck C, Gershenzon J, Haas H, Brakhage AA: The MAP kinase MpkA controls cell wall integrity, oxidative stress response, gliotoxin production and iron adaptation in Aspergillus fumigatus. Mol Microbiol 2011, 82:39-53.

9. Scharf DH, Remme N, Heinekamp T, Hortschansky P, Brakhage AA, Hertweck C: Transannular disulfide formation in gliotoxin biosynthesis and its role in self-resistance of the human pathogen Aspergillus fumigatus. J Am Chem Soc 2010, 132:10136-10141.

10. Arnaud MB, Cerqueira GC, Inglis DO, Skrzypek MS, Binkley J, Chibucos MC, Crabtree J, Howarth C, Orvis J, Shah P, Wymore F, Binkley G, Miyasato SR, Simison M, Sherlock G, Wortman JR: The Aspergillus Genome Database (AspGD): recent developments in comprehensive multispecies curation, comparative genomics and community resources. Nucleic Acids Res 2012, 40:D653-D659.

11. Gilsenan JM, Cooley J, Bowyer P: CADRE: the Central Aspergillus Data REpository 2012. Nucleic Acids Res 2011, 40:660-666. 
12. Gibbons JG, Beauvais A, Beau R, McGary KL, Latgé J, Rokas A: Global transcriptome changes underlying colony growth in the opportunistic human pathogen Aspergillus fumigatus. Eukaryot Cell 2012, 11:68-78.

13. Wang B, Guo G, Wang C, Lin Y, Wang X, Zhao M, Guo Y, He M, Zhang Y, Pan L: Survey of the transcriptome of Aspergillus oryzae via massively parallel mRNA sequencing. Nucleic Acids Res 2010, 38:5075-5087.

14. Feng L, Liu H, Liu Y, Lu Z, Guo G, Guo S, Zheng H, Gao Y, Cheng S, Wang J, Zhang $K$, Zhang Y: Power of deep sequencing and agilent microarray for gene expression profiling study. Mol Biotech 2010, 45:101-110.

15. Wang Z, Gerstein M, Snyder M: RNA-Seq: a revolutionary tool for transcriptomics. Nat Rev Genet 2009, 10:57-63.

16. Marioni JC, Mason CE, Mane SM, Stephens M, Gilad Y: RNA-seq: an assessment of technical reproducibility and comparison with gene expression arrays. Genome Res 2008, 18:1509-1517.

17. Bruns S, Seidler M, Albrecht D, Salvenmoser S, Remme N, Hertweck C, Brakhage AA, Kniemeyer O, Mueller FC: Functional genomic profiling of Aspergillus fumigatus biofilm reveals enhanced production of the mycotoxin gliotoxin. Proteomics 2010, 10:3097-3107

18. Nishida H, Motoyama T, Yamamoto S, Aburatani H, Osada H: Genome-wide maps of mono- and di-nucleosomes of Aspergillus fumigatus. Bioinformatics 2009, 25:2295-2297.

19. Ghazalpour A, Bennett B, Petyuk VA, Orozco L, Hagopian R, Mungrue IN, Farber CR, Sinsheimer J, Kang HM, Furlotte N, Park CC, Wen P, Brewer H, Weitz K, Camp DG, Pan C, Yordanova R, Neuhaus I, Tilford C, Siemers N, Gargalovic P, Eskin E, Kirchgessner T, Smith DJ, Smith RD, Lusis AJ: Comparative analysis of proteome and transcriptome variation in mouse. PLOS Genet 2011, 7:e1001393.

20. Glaesener S, Honecker F, Veltman IM, Gillis AJM, Rohlfing T, Streichert T, Otto B, Brummendorf TH, Looijenga LHJ, Bokemeyer C, Balabanov S: Comparative proteome, transcriptome, and genome analysis of a gonadal and an extragonadal germ cell tumor cell line. J Proteome Res 2008, 7:3890-3899.

21. Vödisch $M$, Albrecht $D$, Lessing F, Schmidt AD, Winkler R, Guthke $R$, Brakhage AA, Kniemeyer O: Two-dimensional proteome reference maps for the human pathogenic filamentous fungus Aspergillus fumigatus. Proteomics 2009, 9:1407-1415.

22. Priebe $S$, Linde J, Albrecht D, Guthke R, Brakhage AA: FungiFun: a web-based application for functional categorization of fungal genes and proteins. Fungal Genet Biol 2011, 48:353-358.

23. Sanchez JF, Somoza AD, Keller NP, Wang CCC: Advances in Aspergillus secondary metabolite research in the post-genomic era. Nat Prod Rep 2012, 29:351.

24. Shi L, Reid LH, Jones WD, Shippy R, Warrington JA, et al: The MicroArray Quality Control (MAQC) project shows inter- and intraplatform reproducibility of gene expression measurements. Nat Biotechnol 2006, 24:1151-1161

25. Gehrke A, Heinekamp T, Jacobsen ID, Brakhage AA: Heptahelical Receptors GprC and GprD of Aspergillus fumigatus are essential regulators of colony growth, hyphal morphogenesis, and virulence. Appl Environ Microbiol 2010, 76:3989-3998.

26. Albrecht $D$, Guthke $R$, Brakhage AA, Kniemeyer O: Integrative analysis of the heat shock response in Aspergillus fumigatus. BMC Genomics 2010, 11:32.

27. Foss EJ, Radulovic D, Shaffer SA, Ruderfer DM, Bedalov A, Goodlett DR, Kruglyak L: Genetic basis of proteome variation in yeast. Nat Genet 2007, 39:1369-1375.

28. da Silva Ferreira ME, Kress MRVZ, Savoldi M, Goldman MHS, Härtl A, Heinekamp T, Brakhage AA, Goldman GH: The akuB ${ }^{\mathrm{KU} 80}$ mutant deficient for non homologous end joining is a powerful tool for analyzing pathogenicity in Aspergillus fumigatus. Eukaryot Cell 2006, 5:207-211.

29. Bentley DR, Balasubramanian S, Swerdlow HP, Smith GP, Milton J, Brown CG, Hall KP, Evers DJ, Barnes CL, Bignell HR, Boutell JM, Bryant J, Carter RJ, Keira Cheetham R, Cox AJ, Ellis DJ, Flatbush MR, Gormley NA, Humphray SJ, Irving $L$, Karbelashvili MS, Kirk SM, Li H, Liu X, Maisinger KS, Murray LJ, Obradovic B, Ost T, Parkinson ML, Pratt MR, Rasolonjatovo IMJ, Reed MT, Rigatti $R$, et al: Accurate whole human genome sequencing using reversible terminator chemistry. Nature 2008, 456:53-59.

30. Trapnell C, Pachter L, Salzberg SL: TopHat: discovering splice junctions with RNA-Seq. Bioinformatics 2009, 25:1105-1111.

31. Mortazavi A, Williams BA, McCue K, Schaeffer L, Wold B: Mapping and quantifying mammalian transcriptomes by RNA-Seq. Nat Methods 2008, 5:621-628.
32. Hardcastle TJ, Kelly KA: baySeq: empirical Bayesian methods for identifying differential expression in sequence count data. $B M C$ Bioinforma 2010, 11:422.

33. Stanke M, Keller O, Gunduz I, Hayes A, Waack S, Morgenstern B: AUGUSTUS: $a b$ initio prediction of alternative transcripts. Nucleic Acids Res 2006, 34(Web Server issue):W435-W439.

34. Vödisch M, Scherlach $K$, Winkler R, Hertweck C, Braun $H$, Roth M, Haas $H$, Werner ER, Brakhage AA, Kniemeyer O: Analysis of the Aspergillus fumigatus proteome reveals metabolic changes and the activation of the pseurotin A biosynthesis gene cluster in response to hypoxia. J Proteome Res 2011, 10:2508-2524.

doi:10.1186/1471-2164-13-519

Cite this article as: Müller et al:: Comparison of transcriptome technologies in the pathogenic fungus Aspergillus fumigatus reveals novel insights into the genome and MpkA dependent gene expression. BMC Genomics 2012 13:519.

\section{Submit your next manuscript to BioMed Central and take full advantage of:}

- Convenient online submission

- Thorough peer review

- No space constraints or color figure charges

- Immediate publication on acceptance

- Inclusion in PubMed, CAS, Scopus and Google Scholar

- Research which is freely available for redistribution

Submit your manuscript at www.biomedcentral.com/submit
C Biomed Central 